\title{
Proceedings of the Society of British Neurological Surgeons with the New England Neurosurgical Society, London, September 1991
}

RADICAL EXCISION OF CRANIOPHARYNGIOMA BY THE TEMPORAL ROUTE

L Symon. National Hospital, London

Craniopharyngioma remains a difficult management problem. The central situation and difficult texture of the tumour, its adhesion to neighbouring blood vessels and basal cranial nerves, and its capacity to wander through more than one intracranial compartment has often resulted in years of progressively impaired health in a patient punctuated by attempts at partial removal, each one frequently leading to further deficit. Attempted radical excision of craniopharyngioma have been made increasingly possible by progress in neuroradiological imaging and the use of microscopic technique.

Between 1977-91 fifty four patients in the National Hospital for Neurology and Neurosurgery have had radical surgical excision of craniopharyngioma by the temporal route where the configuration of the tumour seemed appropriate for this approach. Surgical mortality was $4 \%$. Forty two patients $(78 \%)$ remain well on an average follow up of thirty months. Twelve per cent had major complications: hypothalamic damage, subdural haematoma, scalp collections requiring shunt drainage, and there have been four recurrences in patients where the initial operations have been considered to be microscopically complete.

THE GOOD, THE BAD, AND THE UGLY: TREATMENT STRATEGIES IN THE MANAGEMENT OF CRANIOPHARYNGIOMA

R Hayward, U Andar, W Harkness. Great Ormond Street, London

There is little argument about the ideal treatment of craniopharyngioma. The tumour is benign and non-invasive and the best prognosis should therefore follow a complete removal. The technical difficulties, however, involved in carrying out this strategy make management policies controversial, as can be seen by the number of articles devoted to the subject in the neurosurgical literature, with estimates of complete removal ranging from $\mathbf{7 \%}$ (for transphenoidal surgery) upwards. By analysing a variety of risk factors, it is possible to prescribe for each patient a treatment policy, both surgical and non-surgical, which should provide them with the lowest immediate mortality and morbidity as well as allowing them the most favourable longterm outlook.

Analysis of all the $\mathbf{3 0}$ new cases of craniopharyngioma seen over a ten year period demonstrates how it is now possible, on the basis of both clinical and imaging information (particularly MRI) to determine preoperatively: the scale of the operation that it is safe to perform, the operative approach (such as, subfrontal, temporal, cranio-facial) and the likely requirement for post-operative radiotherapy and the most suitable method for its delivery (conventional, stereotactic or interstitial).
THE TREATMENT OF ACTH SECRETING PITUITARY ADENOMATA BY TRANSPHENOIDAL SURGERY GS Cruickshank, GM Teasdale. Glasgow

A recent British study ${ }^{1}$ on the care of Cushings Disease, cast doubt on the relationship between the type of operation, the pathological findings and the outcome. A total of 84 consecutive patients were reviewed with Cushings disease or Nelsons syndrome. The aims were to determine the accuracy of pre-operative investigations, the operative findings and to relate those with the specific surgical technique (complete selective adenomectomy, incomplete selective adenomectomy, radical subtotal resection, total hypophysectomy) and outcome.

Seventy two patients with pituitary driven Cushings disease, and 12 patients with Nelsons syndrome had transphenoidal pituitary exploration. A discrete macro or microadenoma was located in 60 patients and was judged to be completely removed in 47 . In each case the surgical diagnosis was confirmed by histology and subsequent remission.

Patients without a discrete tumour had either radical subtotal (19) or total hypophysectomy (6). Four of these had a microadenoma. In total 64 patients had an adenoma confirmed by histology, five glandular hyperplasia and in 15 the gland appeared normal. Of those patients with a discrete lesion seen at operation, only $27 / 60(45 \%)$ were correctly predicted by CT scanning and only $6 / 12$ by MRI. Petrosal venous sampling was accurate at confirming a pituitary source for ACTH over-production, but poor at identifying the pituitary localisation of the adenoma. Every patient who had a selective adenomectomy went into remission, these patients recovered their pituitary reserve more rapidly and frequently, than after other operations. Nineteen patients had radical subtotal excision, 11 remitted, and of six patients who had a total hypophysectomy three remitted. Apparent reasons for failure to remit included a missed diagnosis of ectopic ACTH production in two patients and obviously invasive adenoma in a number of patients.

Radiological studies were unable to reliably identify the precise site of a pituitary micro-adenoma. When the operative findings indicated that selective but complete microadenomectomy was feasible, there was a very high prospect of remission and of return or retention of pituitary function and that this remains the optimum treatment for $\mathrm{ACTH}$ producing adenoma.

1 Burke CW, Adams CBT, Esiri MM, Morris C, Bevan JS. Clinical endocrinology 1990;33: 525-37.

CURRENT STATUS OF BORON NEUTRON CAPTURE THERAPY (BNCT) FOR INTRACRANIAL TUMORS H Hatanaka, R Fairchild, D Joel, D Slatkin, J Coderre, WH Sweet. Boston, USA

Hatanaka's group in Japan have four patients, given BNCT using $\mathrm{B}_{12} \mathrm{H}_{11} \mathrm{SH}$, who are working productively as of this year, one having survived for 19 years with a glioblastoma grade IV, despite all eight of the major prognostic criteria being in the adverse category. The authors were unaware of any other such case in the world literature. The other three Japanese patients with gliomas Grades II and III are active after BNCT for 14,13 and 10 years.

Further progress includes completion of reactor portals for a high flux of epithermal neutrons to achieve adequate depth doses (Brookhaven National Laboratory [BNL] and Massachusetts Institute of Technology [MIT]). Definitive design of two other reactors for BNCT at the Idaho Engineering Laboratory [INEL] and Petten, Holland include a striking reduction in undesirable radiation not selective for tumour. Three much improved methods have been developed for quantitative analysis of tissue samples for boron and for precise identification of the organelles within and around the cells of all types.

The securing of massive amounts of pharmacokinetic data from each patient will be possible with the greatly modified General Electric magnetic resonance scanner which depicts ${ }^{11} \mathrm{~B}$ concentrations in small areas throughout the intracranial cavity.

Perhaps the most important progress has been the achievement at Brookhaven of long survival of frontal gliosarcoma in six of 10 rats given $\mathrm{B}_{12} \mathrm{H}_{11} \mathrm{SSH}_{11} \mathrm{~B}_{12}$ as the boron carrier for the BNCT. Long survival was also achieved in seven of 16 rats using boronophenylalanine. These are the first critically controlled studies to produce high percentages of probable cure of gliomas in any species and are perhaps the most crucial favourable biological information relevant to BNCT obtained to date.

TOTAL THORACIC VERTEBRECTOMY FOR PRIMARY MALIGNANT DISEASE BY POSTERIOR APPROACH-A CASE REPORT

G Findlay, P Eldridge. Liverpool

Considerable controversy continues to exist regarding the ideal operative approach for many spinal problems, including tumour. In the high thoracic region, an anterior approach is usually felt necessary to provide safe access for anterior disease. Subsequently a further posterior procedure may be necessary for stabilisation.

An unusual case was presented of primary bone tumour (probable chondrosarcoma) which presented as an exophytic mass in the paravertebral region: involvement of anterior and posterior elements of two vertebrae and ribs on one side.

Resection of tumour gave adequate access to allow total vertebrectomy grafting and Hartshill rectangle fusion at one procedure, achieved from a posterior approach. This was achieved at a high thoracic level where the great vessels made a trans-thoracic procedure more hazardous.

An excellent recovery occurred although unfortunately the malignant nature of the tumour makes a good prognosis unlikely. A 
posterior approach may be possible by individual anatomy and operative approaches tailored to individual cases rather than dictated by convention.

RECENT EXPERIENCE WITH HEMISPHRECTOMY CE Polkey. Maudsley Hospital, London

A personal series was reviewed of 28 hemispherectomies for intractable epilepsy, carried out since 1978. Four were subtotal hemispherectomies; the remaining 24 were total hemispherectomies using Adam's modification. ${ }^{1}$ Follow up was available for 17 patients operated on at least two years before (range 2-14), and using Engels's outcome scale. $^{2}$ Thirteen patients $(76 \%)$ were seizure free or virtually so, two were improved, one was not and one patient died. Four of the patients undergoing total hemispherectomy required CSF shunts from the remaining ventricle, two in the immediate post-operative period. There were no instances of late delayed bleeding although many patients showed asymptomatic dilatation of the remaining ventricle and midline shift. In two cases there were unexpected neurological consequences.

The pathology in the specimens showed 14 examples of Rasmussen's encephalitis, four cases with cortical neuronal migration defect (hemimegalencephaly) and 10 with other pathologies.

\section{Adams CBT. I Neurol Neurosurg Psychiatry 1983;46:617-9. \\ 2 Engel J. Outcome with respect to epileptic seizures. In: Engel J, ed. Surgical treatment of the epilepsies. New York, Raven Press, 1987:553-71.}

HIPPOCAMPAL COMMISSUROTOMY AT THE TIME OF CORPUS CALLOSUM SECTION FOR INTRACTABLE EPILEPSY

DW Roberts, AG Reeves, RE Nordgren, MS Gazzaniga.

The hippocampal commissure, a structure of unclear significance in the human, is conventionally divided at the time of section of the overlying posterior corpus callosum. The benefit in terms of improved seizure control and the risk in terms of cognitive impairment are not well understood. Should the commissure's division accompany corpus callosum section?

Three sets of data were studied for this purpose. Firstly, 98 patients had undergone corpus callosum section. Of these, 27 patients had combined posterior corpus callosum and hippocampal commissure section only when seizure control was suboptimal following anterior corpus callosum section. The percentage of these patients obtaining $80-100 \%$ reduction in generalised seizures approximately doubled following the second surgery ( $29 \%$ versus $62 \%$ ), but correlation of response to extent of initial section was only moderate and distinction between effect of corpus callosum versus hippocampal commissure section was not possible. The second data set was that of EEG cross-correlation and autoregression studies that suggested a diminished role of the hippocampal commissure, compared with the corpus callosum, in seizure propagation. The third area of interest was that of neuropsychological studies that demonstrated impairment of recall but not recognition memory following division of the posterior corpus callosum and hippocampal commissure.
While these lines of evidence are not conclusive, they suggested that in selected patients, particularly those without complex partial seizure manifestations and those in whom protection of memory is a high priority, sparing of the hippocampal commissure at the time of posterior corpus callosum section may be warranted.

HINDBRAIN HERNIA: PRESENTATION, MANAGEMENT AND OUTCOME

PO Byrne, BN Williams. Midland Centre, Warley

A review of 100 patients with symptomatic hindbrain hernia, also known as Chiari Type I malformation, was presented. These cases were selected for being adults with no obvious cause of the hindbrain hernia such as intracranial tumour and also because they had been treated by craniovertebral decompression.

Symptoms and signs on presentation were analysed as "motor", "sensory", "bulbar" and "other" groups. Serious difficulties were encountered in the assessment of the results of management in this condition. Post-operatively, $76 \%$ of the patients were clinically improved, $18 \%$ were stable and $6 \%$ were worse following craniovertebral decompression. There was little correlation between outcome clinically and post-operative imaging. Four patients required additional procedures aimed at improving residual syrinx cavities. The details of the operative approach were presented.

BRAINSTEM DECOMPENSATION IN PATIENTS WITH CHIARI DEFORMITIES AND THE RELATIONSHIP TO CSF VOLUME

EG Fischer. Boston

A patient was reported with a shunt-induced Chiari I deformity and who developed symptoms of pronounced brainstem dysfunction when a large bifrontal craniectomy defect was created. The symptoms were relieved when the shunt, originally placed because of postoperative CSF rhinorrhea, was ligated.

The author suggested that if CSF volume affects the rostro-caudal position of the cerebellar tonsils in relation to the foramen magnum, buoyancy of the brain and reduction in CSF volume may be important factors in the development of a Chiari deformity following insertion of a lumbar shunt. Both factors may be responsible for the caudal migration of the hindbrain seen following ventricular shunts which has been ascribed to cephalo-cranial disproportion. If the hindbrain can be displaced caudally at the foramen magnum when atmospheric pressure is transmitted to the frontal lobes through craniectomy defect, brainstem decompensation in infants with Chiari II malformation may be explained by the effect of atmospheric pressure transmitted to the frontal lobes through the open anterior fontanelle.

THE NATURAL HISTORY OF DIASTEMATOMYELIA UB Andar, RD Hayward, WFJ Harkness. Great Ormond Street, London

Diastematomyelia (57 cases over 12 years) accounted for about a quarter of the cases with spinal disorders seen by the authors.
To decipher its natural history, cases seen early and treated conservatively were compared with patients who had undergone early surgery. Many children had no neurological signs at birth or at time of referral. Some had spinal or limb deformities with associated dysraphic features. After long follow up few were left without any neurological deficits. The problems of prediction of natural history and timing of surgical intervention provoked a vigorous debate.

HARVEY CUSHING'S BRIGHAM HOSPITAL-1991

J Shillito. Boston, USA

In $1932 \mathrm{Dr}$ Harvey Cushing retired as Chief of Surgery at the Peter Bent Brigham Hospital, Boston. The retirement age of 63 years had been established by Dr Cushing and his counterpart in medicine, Dr Henry Christian, when the hospital was opened in 1913.

It was apparently quite a shock to $\mathrm{Dr}$ Cushing that this time arrived so quickly. He returned from a trip to Europe to find that his successor, Dr Eliot Cutler, whom he had trained, was already physically established in the Chief's office, and Dr Cushing's possessions had been removed elsewhere. The Department of Surgery became a very general surgical department, and the number of neurosurgical operations per year dropped precipitously. Dr Cutler maintained that he could operate equally well "in the body cavity" and he assumed responsibility not only for all the intracranial operations but the cardiac operations as well. Dr Horrax, Cushing's longstanding associate, went to the nearby Deaconness Hospital with the Lahey Clinic. Neurosurgery at the Children's Hospital, across the street from the Brigham, continued after Cushing's department under Dr Franc Ingraham, a pupil of Cushing's who had been assigned to set up a pediatric neurosurgical department in 1929 .

When World War II took many surgeons away from the Brigham, including Eliot Cutler, Dr Ingraham also covered the neurosurgical activities at the Peter Bent Brigham Hospital as well as the Children's Hospital. This arrangement continues to the present day. With the help of several now prominent fellows, and his ultimate associate, Dr Donald Matson, neurosurgery at the Brigham was returned to the hands of neurosurgeons.

Dr Ingraham retired as Chief of Neurosurgery in 1964 and died of a myocardial infarction in 1965. Dr Matson continued as Chief but succumbed in 1969 to JacobCreutzfeldt Disease. Neurosurgery was administered temporarily by the author with the help of several other former residents and associates until 1971, at which time it came under the direction of Dr Keasley Welch. After his retirement Dr Peter Black became the Ingraham Professor. Neurosurgical activity now exceeds that of Harvey Cushing's day.

Dr Cushing not only left a remarkable heritage, but he also left some close associates. Adolph Watska, his operating room orderly, continued to work at the Brigham until his death in 1953. Mildred Codding, Cushing's medical artist, continued to work well past her official retirement, until 1980 . She lives on Cape Cod, enjoying her memories and her water colours of the New England scene. 
NEUROSURGICAL MANPOWER AND ACTTVITY SURVEY: 1988-1990

AA Kemeny, PJ Hamlyn. Sheffield, and St. Bartholomew's Hospital, London

The activity and manpower of all neurosurgical units in the UK and the Republic of Ireland for 1988 and 1989 were reported. The 132 consultant posts provided 1179 sessions, of which 88 were academic. Thei age was given by 112 consultants: only 13 were over 60 years of age. There were 33.5 senior registrars and 20 were due for accreditation by mid 1991 . There were 106 registrars: of the 69 clinical posts 33 were occupied by overseas trainees. Whilst half the units have three consultants, $25 \%$ have less. $36 \%$ have no senior registrar and $14 \%$ of units have no UK clinical trainee at any level. This contrasts with a fifth of units having more than two UK clinical trainees.

There was no significant difference in performance between large and small units, although the range was more varied in the latter. The following activity data represent the means and ranges. Admissions per unit, 1225 (469-3200); operations per unit, 800 (157-1600); operations per admission, 0.67 (0.36-1.3); operations per consultant, 242 (86-508); bed numbers, $40 \cdot 3$ (14-100); beds per consultant, $12.8(4 \cdot 7-22)$; admissions per bed per year, $30.4(17 \cdot 8-70 \cdot 2)$; operations per bed per year, $19.8(11.4-45.3)$

One third of units had no designated beds for ventilation and one quarter had beds closed. Forty per cent refused admission of ventilated cases and one quarter did so monthly. Half of these units were outside London. In spite of a $2.5 \%$ nationwide reduction in bed numbers, there was a $6.5 \%$ increase in the total operations from 1988 to 1989.

ALLEGED PROFESSIONAL NEGLIGENCE INVOLVING NEUROLOGICAL AND NEUROSURGICAL PATIENTS: A REVIEW OF 115 CASES

PJEM Wilson. Swansea

Medical negligence litigation is a growth industry for attorneys, while for doctors it is perhaps the most rigorous form of audit. Lawyers will increasingly require us to scrutinise the practices of our peers and thus, indirectly, our own; a task seldom other than instructive.

In the last 115 cases in which the author, as a neuro-clinician, was asked to provide "plaintiff" expert reports, the largest "defendant" group, unsurprisingly, comprised neurosurgeons (42). Orthopaedists (17), casualty officers (13), and general physicians (9) followed.

In the neurosurgical group, spinal disasters exceeded cranial by 2 to 1 . Commonest were, tetraplegia after cervical discectomy or decompressive laminectomy; mishaps of lumbar disc surgery; and major wound sepsis. Cranial problems related mainly to bad outcome with benign tumours. In 15 cases the nexus of causation was very doubtful. Only two cases seemed in essence indefensible. Importantly, only a handful of neurosurgical accidents occurred at the forefront of "high technology." Most were in the setting of commonplace routine.

Among referring specialties there predominated the age-old and seemingly ineradicable tragedies of neglected extradural haematoma; aneurysmal subarachnoid haemorrhage mistaken for migraine; benign spinal tumours labelled as multiple sclerosis; and tardy perception of cord and cauda equina compression.

STEREOTACTIC BIOPSY OF CEREBRAL LESIONS IN PATIENTS WITH AIDS

A Sofat, MF Pell, DGT Thomas, IR Whittle. London and Edinburgh

The CNS is affected in up to $50 \%$ of patients with AIDS. CT scanning can identify cerebral lesions but cannot distinguish toxoplasmosis from other AIDS-related lesions.

Between May 1987 and November 1990 11 patients had stereotactic biopsy. Six of these patients had previously been empirically treated for toxoplasmosis. Histologica diagnosis of the biopsy specimens showed multifocal leucoencephalopathy in six cases, toxoplasmosis in three cases, lymphoma in one case and non-specific changes with hypercellular cerebral white matter and increased astrocytic cells in one case. One patient died and another had a transient worsening of his previous hemiparesis.

CT guided sterotaxy is a reliable and accurate method of obtaining a tissue diag nosis, but the experience of the authors suggests that it is associated with a higher mortality and morbidity than in non-AIDS patients. It is recommended that when neurological signs and symptoms develop in patients with AIDS and a lesion is identified on CT or MRI scanning the patient should receive at least two weeks of anti-toxoplasma therapy. Biopsy should be reserved for those patients with atypical presentation, negative serology or progressive clinical deterioration.

MENINGIOMAS-HORMONE THERAPY IS AN ALTERNATIVE TO SURGERY

C Davis. Preston

A review of surgical mortality and morbidity for most benign and malignant intracranial tumours over the last 15 years reveals a marked improvement, except in the case of meningiomas where operative morbidity and mortality was $20 \%$ with a $25 \%$ recurrence rate.

A series of eight patients (representing a quarter of patients referred from 1988-1990) was presented. These patients were treated for 3 to 38 months (average 24 months) with Gestrinone, an anti-progesterone synthetic steroid. This selected group of patients were monitored clinically, neurophysiologically and with accurate tumour volume estimation on CT. The results showed no significant tumour growth in any of these cases in the medium term. It was suggested that hormone therapy may be an alternative to surgery for meningiomas and was worthy of a rigorous controlled trial.

A QUANTTTATIVE ASSESSMENT OF BRAIN TUMOUR ULTRASONIC ENHANCEMENT ULTRASTABLE LIPID-COATED MICROBUBBLES AS A CONTRAST AGENT

RS Simon, S-Y Ho, CR Perkins, JS D'Arrigo. Farmington, USA

The authors developed lipid-coated microbubbles that were stable for months in vitro and had a narrow range of diameters, from
0.8 to $4.5 \mu \mathrm{m}$. These ultrastable microbubbles were used as an ultrasonic contrast agent in the brain, causing increased intensity of echoes persisting for many hours. Intravenously administered lipid-coated microbubbles accumulated selectively in rat brain gliomas, exhibiting enhancement of echogenicity for up to one hour. With this contrast, growing lesions could be imaged two days earlier than without contrast. Using a lipidspecific stain, the distribution of the bubbles in the tumour and throughout the brain was characterised.

On the scan, the enhancement of the tumour was accompanied by a change in the signal-to-noise ratio of the echoes from the tumour. Using spectral analysis characteristic textural changes were associated with contrast-enhanced tumour. Lipid-coated microbubbles could target other brain tumours in addition to gliomas; specifically Walker tumour and melanoma metastases in the rat brian were identified on the scan using lipidcoated microbubble contrast. Lipid-coated microbubbles, when injected intravenously, intensified the ultrasonic echoes from a tumour lodged in subcutaneous tissue (Walker-256 carcinosarcoma). The enhancement of the tumour remained for 15-30 minutes. Oil-Red-O lipid stain identified the microbubbles in the tumour area but not in the adjacent soft tissue. There was a significant change of signal-to-noise ratio associated with the echo enhancement.

Finally, the bubbles could be made to resonate, causing a bubble-tissue energy exchange (cavitation). Cavitation causes tissue destruction in other biological systems In this context, we will present our preliminary data for therapeutic applications of the lipid-coated microbubbles.

IMAGING OF AXONAL TRANSPORT: IS THE AXOPLASMIC FLOW CLINICALLY RELEVANT? AG Filler, BA Bell, FA Howe, JR Griffiths, M Flowers, H Sharma, HR Winn, TW Deacon. London, Seattle and Boston, USA

Many neurosurgical and neurological diseases cause disturbances in the natural transport of molecules and vesicles along axons. However, because these disturbances of transport have not been directly detectable in living humans or intact animals, little is known about their severity. Because the dynamics of disturbed axoplasmic flow in nerve compression and neuropathy has been unknown, it has never figured prominently in models of clinical problems.

A series of advances in the laboratory has now led to preliminary success in developing axonal transport tracer compounds which can be detected by MRI and by PET. After intramuscular injection of a ferrite-based tracer, MRI show a marked contrast change in the nerve supplying that muscle reflecting a $50 \%$ reduction in $\mathrm{T} 2$.

For this project, a new kind of PET tracer had been developed with transition metal positron emitting nuclei included in spine ferrite crystals. These "spinel moderated positron emitters" reduce tissue ionizations, improve image resolution, and increase the intensity of the PET signal. In initial tests a nerve gel phantom $1 \mathrm{~mm}$ in diameter was detected using a low resolution multiwire proportional PET camera.

It is expected that these new agents will be useful in locating and in confirming suspected sites of nerve injury, testing for 
transport across sites of spinal cord compression, distinguishing nerve crushes from nerve lacerations, and in the demonstration of a variety of other pathologies previously inaccessible to neuroradiological detection.

RESULTS OF MICROVASCULAR DECOMPRESSION, OPEN RHIZOTOMY, RADIOFREQUENCY LESION AND NEURECTOMY IN 332 PATIENTS WITH TIC DOULOUREUX: ARE THESE DENERVATION OPERATIONS?

EC Tarlov. Burlington, MA, USA

The mechanisms by which relief of tic douloureux occurs after surgery have been much discussed. Cogent criticisms of the Janetta vascular decompression theory have been raised by Adams. The practical matter is that tic pain can be relieved, often lastingly, by a variety of operations. Whether they share a common mechanism has not been established.

Three hundred and thirty two patients with medically intractable tic douloureux were treated surgically between June 1982 and June 1988. Ninety one had microvascular decompression; 58 patients in whom no vascular compression was found were treated by trigeminal rhizotomy of 30-100\% of the sensory root. The operative findings at open operation were categorized in five sub groups: no vascular contact at trigeminal nerve root entry zone, artery touching root, artery indenting root, artery wedged between root entry zone and brainstem, and venous contact. Seven patients had infraorbital neurectomy, five had treatment of mass lesions and 148 had radiofrequency lesions. Of this total group, during 1982 to 1989 there were seven recurrences among the microvascular decompression group (7/91), none recurred among the neurectomy group (0/7), two recurred among the rhizotomy group (2/58), none recurred among the mass lesion group $(0 / 5)$, and 18 recurred among the 148 radiofrequency lesion group (18/148).

The entire group was followed up by questionnaire two to nine years postoperatively to evaluate long term relief of tick pain, late recurrence of tic pain, annoying sensory loss, anesthesia or hypesthesia dolorosa and other symptoms. The author supports the hypothesis that denervation of the trigeminal system accounts for the relief of pain afforded by all of these procedures.

REACTIVITY OF THE RAT MIDDLE CEREBRAL ARTERY IN CONTROLS AND FOLLOWING ISCHAEMIA-A PRELIMINARY REPORT

RJ Laing, A Stawowy, J Warnke, J Jakubowski. Sheffield

Little is known about the reactivity of cerebral blood vessels after a period of acute ischaemia. Ischaemia might damage the muscle cells or the endothelium or both Longa's model ${ }^{1}$ was used to produce a focal ischaemia in the MCA territory. The severity of ischaemia was evaluated by measuring cerebral blood flow (CBF) using the hydrogen clearance technique. Following four hours of ischaemia the animal was killed the MCAs prepared for myography. The contractility of the arteries to potassium and prostaglandin F2A was determined. The control group comprised 21 animals with mean CBF 87+/- $10 \mathrm{ml} / 100 \mathrm{gm} / \mathrm{min}$ in the MCA territory. The preparations remained stable for up to eight hours. In ten animals severe ischaemia was produced with mean
CBF $17+/-7$ in the MCA territory and was maintained for four hours. In MCAs from the ischaemic area the preliminary results suggested that contraction in response to potassium was preserved $(1.8+/-0.36 \mathrm{mN} /$ $\mathrm{mm}$ in control and $1.7+/-1.0 \mathrm{mN} / \mathrm{mm}$ in ischaemic) but that to $\mathrm{PGF}_{2 a}$ was impaired $(1.0+/-0.25 \mathrm{mN} / \mathrm{mm}$ in control and 0.65 $+/-0.37$ in ischaemic).

These early results were sufficiently encouraging to warrant further studies with particular emphasis on endothelial function which may be more susceptible to ischaemic damage.

1 Longa EL, et al. Stroke 1989;20:84-91.

THE EFFECTS OF HYPOTENSION ON INFARCT SIZE, CEREBRAL OEDEMA AND CEREBRAL BLOOD FLOW AFTER EXPERIMENTAL MIDDLE CEREBRAL ARTERY OCCLUSION

RD Strachan, PJ Kane, JR Chambers, S Cook, AD Mendelow. Middlesbrough and Newcastle

Hypotension after a cerebral ischaemic insult affects clinical outcome in patients with occlusive cerebrovascular disease, subarachnoid haemorrhage or head injury. This study was designed to establish the effect of a short period of modest hypotension on infarct size, cerebral oedema and cerebral blood flow after MCA occlusion in the rat. A new method of closed-loop blood pressure control was presented which allowed a precise reduction in blood pressure by hypovolaemia alone.

Forty four male Wistar rats were studied in six groups. In the hypotensive groups, the blood pressure was reduced to $70 \mathrm{mmHg}$ for 30 minutes immediately after MCA occlusion. Results showed that in the hypotensive animals: Infarct size was larger $\left(190.3 \pm 8.8 \mathrm{~mm}^{3}\right.$ and $230.6 \pm 10.1 \mathrm{~mm}^{3}$; $\mathrm{p}<0.01$ ); cerebral oedema was greater in the cortex (specific gravity $=1.0455 \pm 0.0013$ and $1.0493 \pm 0.0011 ; \mathrm{p}<0.05)$ and postocclusion cerebral cerebral blood flow was less in the lesioned hemisphere $(39 \cdot 1 \pm 2 \cdot 1$ and $27.2 \pm 2.4 \mathrm{ml} / 100 \mathrm{~g} / \mathrm{min} ; \mathrm{p}<0.001)$ and blood flow failed to show the same recovery characteristics after reperfusion.

These studies confirm that modest hypotension after infarction has significant deleterious effects on the extent of ischaemic injury.

CALCITONIN GENE RELATED PEPTIDE INCREASES CEREBRAL BLOOD FLOW IN A MODEL OF FOCAL CEREBRAL ISCHAEMIA?

WAS Taylor, SGC Sydserff, BA Bell. Atkinson Morley's Hospital, London

Calcitonin gene related peptide (CGPR) is an endogenous neuropeptide present throughout the perivascular neural network which is released following intracranial haemorrhage. It may have a protective role via its potent vasodilatory action, and be of use in vasospasm following subarachnoid haemorrhage, were early clinical results have been encouraging. ${ }^{1}$

In this study, anaesthetised male Vistar rats were ventilated at normocapnia, and cerebral blood flow (CBF) was measured by hydrogen clearance. A dose response curve for CGPR was established by infusing 40,80,100 and $120 \mathrm{ng} / \mathrm{kg} / \mathrm{min}$, and no change was found in $\mathrm{CBF}$ until a dose of $120 \mathrm{ng} / \mathrm{kg} / \mathrm{min}$ was reached, when mean arterial pressure fell from $96.4 \pm 3.0 \mathrm{mmHg}$ to $85.1 \pm 2.4$ $(t=3.00, p<0.01)$, and CBF from $91.5 \pm 3.0 \mathrm{ml} / 100 \mathrm{~g} / \mathrm{min}$ to $81.9 \pm 1.8 \mathrm{ml}$ $100 \mathrm{~g} / \mathrm{min}(\mathrm{t}=2.92, \mathrm{p}<0.01)$. Middle cerebral artery (MCA) occlusion was produced using an intraluminal suture technique, ${ }^{2}$ and a dose of $100 \mathrm{ng} / \mathrm{kg} / \mathrm{min}$ was selected for rats undergoing MCA occlusion. Groups of ten rats were infused with either CGPR or normal saline, and in the CGPR group CBF was significantly higher $(85.9 \pm 2.9 \mathrm{ml}$ $100 \mathrm{~g} / \mathrm{min}$ ) when compared to the control group $(63.7 \pm 2.1 \mathrm{ml} / 100 \mathrm{~g} / \mathrm{min} ; t=6.26$ $\mathrm{p}<0.001)$. This increase was maintained throughout the period of ischaemia in both the ischaemic and non ischaemic hemispheres.

These findings suggest that CGPR, in a dose below that which produces hypotension, only affects CBF when an insult to the cerebral circulation has occurred, and may explain its possible efficacy in cerebral vasospasm secondary to subarachnoid haemorrhage. 1 Johnston FG, Bell BA, Robertson IJA, et al. 2 Longa EZ, Weinstein PR, Carlson S, et al. Stroke
1989;20:84-91.

TRANSCRANIAL DOPPLER VELOCITY AND CEREBRAL BLOOD FLOW CHANGES. A SIMULTANEOUS STUDY USING THREE VASODILATORS

JL Martin, S Perry, JD Pickard. Southampton

Uncertainty surrounds the prediction of CBF changes from the analysis of Doppler ultrasound flow velocity changes in basal cerebral arteries. The simultaneous effects of a-CGRP, nimodipine, and glyceryl trinitrate (GTN) on CBF and basilar artery mean Doppler flow velocity in the anaesthetised rabbit model $^{1}$ were presented.

Paired measurements of cortical CBF (hydrogen clearance method) and mean basilar artery Doppler flow velocity ("Transcan" EME, Uberlingen, Germany) were recorded during infusions of: $a$-CGRP iv $(1,5,10,50$, $100 \mathrm{ng} / \mathrm{kg} / \mathrm{min}$.), nimodipine iv $(0.1,0.5,1$ $5 \mu \mathrm{g} / \mathrm{kg} / \mathrm{min})$, and glyceryl trinitrate iv $(1,4$, $8,16,32,64 \mu \mathrm{g} / \mathrm{kg} / \mathrm{min})$. Each dose was infused for fifteen minutes.

$a$-CGRP caused a dose-dependent increase in TMV (from $23 \pm 1 \mathrm{SE}$ to $30 \pm 2 \mathrm{~cm} / \mathrm{s}, \quad \mathrm{p}<0.05$ ), whilst CBF remained unchanged. GTN caused a dopedependent increase in CBF (from $36 \pm 4$ to $65 \pm 10 \mathrm{ml} / 100 \mathrm{~g} / \mathrm{min}, \quad \mathrm{p}<0.05)$, whilst TMV remained unaltered. With Nimodipine TMV increased in a dose dependent manner (from $38 \pm 3$ to $84 \pm 7 \mathrm{~cm} / \mathrm{sec}, p<0.01$ ), and CBF also increased in a dose dependent manner (from $38 \pm 3$ to $95 \pm 6 \mathrm{ml} / 100 \mathrm{~g} / \mathrm{min}$, $\mathrm{p}<0.01)$.

CBF changes cannot be predicted from basal cerebral artery flow velocity changes alone. Increases in flow velocity in one artery supplying the circle of Willis, even if this represents a true increase in volume flow, equally may represent an increase in intracranial to extracranial shunt flow. CGRPinduced flow velocity changes may occur by this mechanism. GTN increased CBF but without any change in flow velocity perhaps reflecting dilatation of the basilar artery as well as dilatation of intracerebral arteries. With nimodipine the changes in CBF paralleled the changes in flow velocity. 1 Nelson RJ, Perry S, Hames A, et al. $\mathcal{J}$ Neurosurg
1990;73:601-10. 
TREATMENT OF EXPERIMENTAL CEREBRAL

VASOSPASM USING THE $1 \mu$ S PULSED DYE LASER

R Macfarlane, A Teramura, NT Zervas. Cambridge, UK and Boston, USA

Laser energy at a wavelength of $480 \mathrm{~nm}$ was applied in $1 \mu \mathrm{us}$ pulses of $3-10 \mathrm{~mJ}$ to two models of vasospasm. Rabbit common carotid arteries $(n=40)$ constricted chronically by the application of human blood within a silicone sheath, and dog basilar arteries ( $n=5)$ subjected to a "two-bleed" subarachnoid haemorrhage protocol, were dilated by endovascular laser therapy from a single quartz fibre. Vessels were restored to near normal diameter, and dilatation persisted for the duration of study. No instances of perforation, arterial thrombosis, or of aneurysm formation were observed for up to 60 days after treatment. Prophylactic pretreatment of normal vessels was able to attenuate the development of vasospasm. This study suggests that the $480 \mathrm{~nm}$ pulsed dye laser may have an application for the treatment or prophylaxis of cerebral vasospasm.

FFFECT OF CALCITONIN GENE RELATED PEPTIDE ON OUTCOME AFTER ANEURYSMAL SUBARACHNOID HAEMORRHAGE

European CGRP in subarachnoid haemorrhage study group

Calcitonin gene related peptide (CGRP) is an endogenous neuropeptide presen throughout the perivascular neural network and is released following subarachnoid haemorrhage $(\mathrm{SAH}) .^{1}$ It is a potent vasodilator, and a pilot study in aneurysmal SAH suggested that it can reverse ischaemic deficits in some patients. ${ }^{2}$ A randomised multicentre open comparison of CGRP against standard best managment in patients suffering ischaemic deficits after surgery for a ruptured intracranial aneurysm has been conducted. Patients aged 18-70 years who developed a focal neurological deficit, or had a reduction of two or more points on the Glasgow coma scale (GCS) after surgery, were entered after a CT scan had excluded non-ischaemic causes for their neurological deficit. Informed consent was obtained and preg nancy, uncorrected hypovolaemia, and serious concomitant illness were exclusion criteria. After randomisation, 62 patients were allocated to receive an infusion of $0.6 \mathrm{ug} / \mathrm{min}$ of CGRP for a minimum of 4 hours up to a maximum of 10 days, and 55 patients received standard best management. GCS and haemodynamic parameters were assessed during the patient's hospital stay, and all patients were followed up at three months by an independent investigator, who was blind to their treatment. Outcome was measured on the Glasgow Outcome Scale.

Sixteen neurosurgical units contributed patients, twelve centres in the UK and Ireland, and four in Europe. Of the 117 patients randomised, 99 met the full inclusion criteria of the study protocol. At three month follow up $66 \%$ of patients treated with CGRP and $59 \%$ of patients receiving best management, had a good recovery, but the difference between the two groups was not significant. Hypotension was a common side effect of the CGRP infusion. Although significant beneficial effect has not been demonstrated by this trial, a clinically useful benefit has not been exluded.

1 Juul R, Edvinsson L, Gisvold SE, et al. $B r f$ Neurosurg 1990;4:171-80.

2 Johnston FG, Bell BA, Robertson IJA, et al. Lancet 1990;335:869-72.

DISORDERED SALT AND WATER REGULATION IN NEUROSURGICAL PRACTICE: MODERN MANAGEMENT PERSPECTIVES OBTAINED FROM RECENT STUDIES Y Lolin, A Jackowski, L Symon. Queen Square, London

In the 1950s hyponatraemia in intracranial disease was felt to represent a derangement in an unknown salt regulating factor with an excessive urinary excretion of sodium. The term cerebral salt-wasting syndrome was coined and patients treated with salt and water replacement therapy. Then in 1957 Schwartz postulated inappropriate secretion of antidiuretic hormone (SIADH) resulting in dilutional hyponatraemia as causing the hyponatraemia observed in two patients with bronchogenic carcinoma; SIADH came to be accepted as the aetiology of the majority if not all cases of hyponatraemia in neurological and neurosurgical practice and fluid restriction became the standard treatment.

The distinction between which of these two mechanisms is responsible for the hyponatraemia so commonly encountered is not just academic as their management differs so markedly. The authors now routinely measure the fractional clearances of sodium and water, and assay plasma renin, aldosterone and $\mathrm{ADH}$ as necessary in hyponatraemi patients suffering from SAH and other intracranial disorders to correctly identify the underlying causation. The results demonstrated that in the majority of patients, hyponatraemia is due to salt-wasting with an increase in free water clearance whilst only a minority are due to SIADH where the free water clearance is decreased. This has enabled us to choose appropriate treatment regimes for individual hyponatraemic patients without the risk of instituting therapy that is at best inappropriate or at worst postively harmful.

ANEURYSMAL SUBARACHNOID HAEMORRHAGE: AN AUDIT OF CLINICAL FEATURES, OUTCOME ASSESSMENT \& COMMUNICATION WTTH GPS KM Morris, MDM Shaw, PM Foy. Liverpool

During 1990 all patients admitted to the Mersey Regional Neurosciences Unit after intracranial haemorrhage were prospectively studied to determine clinical course and outcome at six months. A total of 228 patients were screened; 142 had proven aneurysmal subarachnoid haemorrhage (SAH) of whom 128 were medically and neurolgocially suitable for surgery. The management outcome of this group of patients was assessed using the Glasgow Outcome Scale. They attained the following scores at six months: I- $64 \cdot 8 \%$, II $-12 \cdot 7 \%$, III- $7 \cdot 7 \%$, IV-0\%, V-14.8\%

Management outcome was assessed by General Practitioners and compared with case note reports and a single neurosurgical observer. Surprisingly $10 \%$ of GPs had never received a typed discharge summary. Of those GPs receiving a summary $96 \%$ felt that these were adequate but $32 \%$ suggested alterations in emphasis and contents to allow them to more easily continue the management of their patients.
INFLUENCE OF SURFACE COATING ON ADHERENCE OF BACTERIA TO SHUNTS

PR Eldridge, J Bridget, J Punt, SP Denyer. Nottingham

Infection remains a significant cause of shunt failure. Effects of such infection, and the need for shunt revision contribute to a high morbidity for this complication. The initial adherence of bacteria to the shunt may be important in the establishment of both colonisation and infection as has been found with intravascular catheters. In these cases, it was noted that coatings designed and applied to avoid thrombosis also decreased bacterial adherence. One such coating is "hydrom" which is a hydrogel material.

An in vitro assessment of this material applied to shunts was presented. Specimens of tubing, coated and uncoated were immersed in bacterial suspensions of varying concentrations for various times. Adherence was assessed by biofluorescence staining with automated counting. Several strains of bacteria were used, principally Staphylococcus epidermis, including isolates from shunt infections. In both kinetic and concentration studies a tenfold advantage of the coated material was demonstrated. Unfortunately, poor bonding between hydromer and shunt make the method currently impractical.

SHUNT IMPLANATATION: TOWARDS ZERO INFECTION DA Lang, G Lena, L Genitori, E Empime, M Choux. Marseille, France

Reduction of shunt infection has important implications for late neurological and intellectual development in children with hydrocephalus. However, infection is the most important complication of shunt implantation in children, and even in the most recent series infection rates range from $2-5 \%$.

In Marseille shunt practice was audited in order to study the shunt infection rate and to determine the risk factors associated with shunt infection. Two groups of patients were studied. From 1975 to 1982,1068 procedures were performed in 517 children. In this group the infection rate was $5.60 \%(10.55 \%$ per patient). A new protocol for shunt implantation was then introduced, comprising step by step modifications to the pre, intra and post operative management of the children and between January 1983 and December 1990 have carried out 1043 procedures. In this group of 617 patients there were two infections. One of these occurred in a new patient and the other after a shun revision. Overall after introduction of the new protocol the infection rate was $0.19 \%$ $(0.32 \%$ per patient).

Shunt infection is an avoidable operative complication and that a $0 \%$ infection rate is a realistic goal. Shunt implantation is a complex procedure and must be done by an experienced neurosurgeon.

A PROSPECTTVE STUDY OF IDIOPATHIC NORMAI PRESSURE HYDROCEPHALUS-GUIDELINES FOR OUTPATIENT INVESTIGATION

JD Pickard, H Newton, A Greene, HK Richards, AH Lovick, DA Lang, RD Hoare. Cambridge, Southampton and London

Elderly patients with possible NPH are fragile and their investigation and management as inpatients may prove protracted, expensive and of uncertain overall benefit. The value of CT, MRI, Cerebral Blood Flow (SPECT; 


\begin{tabular}{llcll}
\hline Predictive Factor & $(n)$ & Sensitivity & Specificity & Accuray \\
\hline No vascular history & $(24)$ & $57 \%$ & $47 \%$ & $50 \%$ \\
CT-gyral atrophy & $(25)$ & $43 \%$ & $44 \%$ & $44 \%$ \\
MR-absent PVL & $(25)$ & $57 \%$ & $89 \%$ & $80 \%$ \\
matter leep white & $(21)$ & $71 \%$ & $86 \%$ & $81 \%$ \\
IPC-B waves ( $>30 \%)$ & $(14)$ & $80 \%$ & $88 \%$ & $79 \%$ \\
-C Cut $_{\text {our }}(\leqslant 0.09)$ & $(22)$ & $100 \%$ & $24 \%$ & $41 \%$ \\
\hline
\end{tabular}

${ }^{133}$ Xenon) and ICP monitoring with CSF outflow conductance measurements in predicting the response to ventriculo-peritoneal shunting was assessed in 25 patients (mean age 73 years; $19 \mathrm{M}$; $6 \mathrm{~F}$ ) with possible idiopathic hydrocephalus. Detailed assessment included psychometric and functional scores (table).

In this age group, patients with idiopathic hydrocepahlus, gait apraxia and slowness of mentation should be considered for shunting where there are no focal deep white matter lesions on MRI ( $71 \%(5 / 7))$ improved for more than one year; revision: 1 ; postoperative fits: 1;). Such white matter lesions do not preclude considerable benefit from shunting in a minority (2 excellent results out of 14 patients-14\%) but further support by 24 hour ICP monitoring and test removal of CSF should be used to justify the risk of surgical intervention in this vulnerable subgroup (postoperatiuve cardiac arrest-resuscitated: 1 ; postoperative hemiparesis: 1 ; infection: 2; fractured femur: 1; subdural haematoma: 1; revision: 4; late strokes (3) and late deaths (5) not necessarily related to operation).

Supported by the Sir Jules Thorn Charitable Trust.

THE PROBLEM OF PARADOXICAL AIR EMBOLISM AND ITS PREVENTION DURING NEUROSURGERY: THE IMPORTANCE OF PREOPERATIVE CONTRAST ECHOCARDIOGRAPHY

MP Roberts, LD Gillam, JS Mikan. Hartford, Con, USA

Air may enter the vascular system during almost any surgical procedure. Air embolisation during neurosurgery is most likely to occur with the patient in the sitting position. With the exception of massive emboli, the air enters the pulmonary vasculature, passes across the alveolar membrane, and is harmlessly expired. Paradoxical embolism takes place when air is shunted from the right to the left heart, typically through a patent foramen ovale. Disastrous injury or death may then result if even a small quantity of air enters the cerebral circulation or coronary arteries.

Two cases of paradoxical air embolism during cervical disc surgery in the sitting position were discussed, one in which the patient was left decerebrate and a second case in which the patient suffered a chronic Parkinsonian syndrome. The techniques used to prevent air embolism and the importance of preoperative contrast echocardiography were detailed.

HAEMODYNAMIC FUNCTIONAL CLASSIFICATION OF CEREBRAL ARTERIOVENOUS MALFORMATIONS USING SINGLE PHOTON EMISSION TOMOGRAPHY (SPET) AHS Huneidi, MJ Carroll, J Bomaji, C Nimmon, J Jenkins, DS Montefiori, PN Plowman, P Hamlyn, KE Britton, F Afshar. St Bartholomew's Hospital, London

Forty five patients, age $35 \pm 2$ yrs $(23 \mathrm{M})$, presented with cerebral haemorrhage and/or epilepsy due to arteriovenous malformations (AVM) as proven by head CT scan and cerebral angiography. They were studied prospectively with dynamic cerebral blood flow and brain SPET, at the same session, using non-diffusible human serum albumin (HSA) labelled with Tc-99m (600 MBq). They were followed up for a mean of $9.5 \pm 1$ months and

Two types of cerebral AVM could be identified: 1) Fast Flow AVM (65\%) with affected to non-affected hemisphere mean transit time ratio of $0.87 \pm 0.02$; and a flow ratio of $1.31 \pm 0.04$; and 2) Slow Flow AVM $(35 \%)$ with a mean transit time ratio of $1 \cdot 10$ \pm 0.03 and a flow ratio of $0.92 \pm 0.06$, which tended to be mainly venous AVM. Both differences were significant for transit time and flow $(p<0.01)$. Studies with HMPAO showed significant perfusion defects, in contrast to HSA-SPET, indicating serious regional malfunction.

In the fast flow AVM group reduction in flow was detected between three and six months after radiosurgery. This contrasts with the minimum twelve month period for angiography. ${ }^{1}$ This assessment may prove of good prognostic value.

\section{Kemeny, A, et al. $\mathcal{f}$ Neurol Neurosurg Psychiatry
1989;52:554.}

SURGICAL OUTCOME WITH ARTERIOVENOUS MALFORMATIONS

AR Aspoas, AD Mendelow, J Arrotegui, A Gholkar. Newcastle

Despite advances with interventional neuroradiology and stereoradiosurgery, surgical excision of arteriovenous malformations (AVM) remains an effective form of treatment, often used in conjunction with these new developments. To balance the hazards of surgery against the natural history of AVM, it is necessary to be able to predict the outcome from surgery (table).

Spetzler ${ }^{1}$ proposed a grading system based on angiographic findings which predicts the operative morbidity and mortality of surgical excision. The results in forty four patients were compared with those of Spetzler to assess the validity of the grading system.

Spetzler grading is useful in quantifying surgical risk. 1 Spetzler RF, Martin NA. $f$ Neurosurg 1986;65:
476-83. had $2.4 \pm 0.2$ studies.
MANAGEMENT OF LIFE-THREATENING ACUTE INTRACEREBRAL HAEMATOMAS DUE TO VASCULAR LESIONS.

D Gentleman, R Bullock. Glasgow

The management of unconscious or deteriorating patients with life-threatening clots and underlying vascular anomalies is still controversial. Even among experienced surgeons attitudes range from therapeutic nihilism to vigorous intervention.

The authors reported their recent experience with 30 patients ( $60 \%$ male, $90 \%$ aged under 50) presented in coma or deteriorated after admission, in whom CT scan showed a massive clot from rupture of a cerebral aneurysm (15 cases) or arteriovenous malformation (AVM) (15 cases). The clots were in the left cerebral hemisphere (14 cases), right hemisphere (15 cases), or cerebellum (one case). All patients had emergency clot evacuation, $90 \%$ within 24 hours of the onset of coma or deterioration, with pre-operative cerebral angiography in 19 cases. At the same operation, 14 of 15 aneurysms were clipped, and six of 15 AVMs were excised. At six months, outcome showed good recovery or moderate disability in 20 cases, five were severely disabled, and five died. Outcome was unaffected by pre-operative coma score or by the hemisphere affected, but patients with AVMs did better (13 of 15 good or moderate) than those with aneurysms (seven of 15 good or moderate).

These results show the value of emergency clot evacuation, with or without definitive surgery, in such desperately ill patients, especially if there is evidence that the underlying lesion is an AVM.

SKULL BASE CHORDOMAS

LD Watkins, ESS Khudados, $M$ Kaleoglu, $H$

Alan Crockard. Queen Square, London

The presentation and results of treatment were reviewed for 45 patients with skull base chordoma presenting between 1958-88. With few exceptions, ${ }^{1}$ previous studies have combined results for clival and sacral chordomas, or for chordomas and other similar tumours such as chondrosarcoma. Analysis of the survival data for our patients suggests that there are two subgroups with distinct survival patterns: one group with high mortality within the first four years, and a second group with an indolent disease process and near normal life expectancy. Survival did not correlate with the year of operation, suggesting that changes in treatment over the period of the study did not distort the results. Series in which conventional radiotherapy has been given to all patients after surgery have not shown greatly improved survival compared with results published 57 years ago for Table Independent $=$ normal/good recovery/minimal or moderate disability; Dependent $=$ severe disability/major
deficit/vegetative

\begin{tabular}{lrrrrrr}
\hline & No & \multicolumn{2}{c}{ Independent (\%) } & \multicolumn{2}{c}{ Dependent (\%) } \\
\hline Grade & P & S & P & S & P & S \\
I & 6 & 23 & 100 & 100 & 0 & 0 \\
II & 8 & 21 & 100 & 100 & 0 & 0 \\
III & 15 & 25 & 93 & 96 & 7 & 4 \\
IV & 9 & 15 & 100 & 93 & 0 & 12 \\
V & 6 & 16 & 67 & 88 & 33 & \\
Total: & 44 & 100 & & & &
\end{tabular}


patients treated by surgery alone. However, proton radiation therapy, has shown very promising results. ${ }^{2}$ A treatment protocol is suggested initially using subtotal excision alone, supplemented with proton radiation in those patients who have early recurrent disease. This treatment protocol could be compared with a matched group for whom proton therapy is used immediately following initial surgery.

1 O'Neill P, Bell BA, Miller JD, et al. Neurosurgery 1985;16:166-70

2 Austin-Seymour M, Munzenrider J, Goitein M, et al. $\mathcal{F}$ Neurosurg 1989;70:13-17.

IMMEDIATE REVERSAL OF "CRUCIATE PARALYSIS" FOLLOWING TRANSORAL REMOVAL OF ODONTOID IN RHEUMATOID ARTHRTTIS WITH BASILAR

INVAGINATION AND CHAIRI I MALFORMATION

G Mohr, R Schondorf, M Black, M Maleki. Montreal, Quebec, Canada

"Cruciate paralysis" described by HS Bell" consists of disproportionate motor deficits involving both upper limbs with relative sparing of the lower limbs and is usually diagnosed initially as central cord syndrome. The currently accepted, although still controversial patho-physiologic mechanisms of this "alternate brachial diplegia" relates to the midline involvement of the more rostally decussating upper limb fibres of both corticospinal tracts at the medullo-cervical junction. This rare entity has been described in upper cervical spine trauma, mainly odontoid fracture-dislocation, rheumatoid arthritis and basilar invagination with cerebellar ectopia.

This 47 year old woman with severe rheumatoid arthritis developed rapidly progressive tetraparesis with complete flaccid paralysis of both upper limbs and less severe involvement of lower limbs as well as severe lower cranial nerve deficits requiring tracheostomy and feeding jejunostomy. Burning dysesthesia and neuralgic pain in trunk and upper extremities were also present. Because of significant medullary compression from odontoid impaction associated with basilar invagination and Chiari I malformation, posterior fossa decompression and lateral occiput-C2 bony fusion were first performed without improvement.

In a second sitting, via transoral transpharyngeal approach, the odontoid process and part of the C2-body were removed using a high-speed diamond drill and the transverse ligament of the axis was divided longitudinally, resulting in bulging of the dura through the bony decompression. Immediately postoperatively, return of motor function of both arms was noted, followed by a rapid normalisation of her gait. The tracheostomy and jejunostomy were removed ten days later after complete restitution of swallowing and speech capacities.

Rapid and complete motor recuperation has consistently been observed in cruciate paralysis and indicates minimal parenchymal damage but important physiological impairment. Another patient with similar symptoms from rheumatoid dens-subluxation and rapid reversal of branchial diplegia after $\mathrm{C} 1-\mathrm{C} 2$ posterior fusion was also presented.

1 Bell HS. $\mathcal{f}$ Neurosurg 1970;33:376-90. THE VALUE OF MONITORING TRANSCRANIAL
DOPPLER AND JUGULAR BULB VENOUS OXYGEN SATURATION DURING INTRACRANIAL PRESSURE THERAPY AFTER SEVERE BRAIN INJURY KH Chan, NM Dearden, S Midgeley, JD Miller. Edinburgh

The role of transcranial doppler (TCD) and jugular bulb venous oxygen saturation (SJO2) in monitoring intracranial pressure (ICP) therapy was examined in 22 patients with severe brain injury undergoing continuous recording of cerebral perfusion pressure (CPP), arterial oxygen saturation and $\mathrm{SJO} 2$, from which the arterial-jugular venous oxygen content difference (AVDO2) could be derived. Continuous recording of middle cerebral artery blood flow velocity and pulsatility index (PI) was performed during changing CPP. Treatment was initiated when ICP remained $22 \mathrm{mmHg}$ or CPP fell below 60 mmHg. Treatment was considered successful only if ICP fell below $20 \mathrm{mmHg}$ and CPP was increased or preserved above 60 $\mathrm{mmHg}$.

Forty nine treatment responses were recorded. Therapy always reduced ICP to some extent. When pretreatment CPP was 60 $\mathrm{mmHg}$ and treatment was successful, CPP change (post-treatment minus pretreatment value) correlated with changes in $\mathrm{SJO} 2$ ( $r=$ $0.678, \mathrm{p}<0.01$ ), AVDO2 ( $\mathrm{r}=-0.69$, $\mathrm{p}<$ $0.01)$ and PI $(r=-0.76, p<0.001)$, indicating global improvement in cerebral oxygen delivery. In contrast, with treatment success at pretreatment CPP $60 \mathrm{mmHg}$, there was no change in SJO2, AVDO2 or PI but CPP increase correlated with increases in mean velocity ( $r=0.501, p<0.05$ ), suggesting intact autoregulation. In seven (all hypnotic therapy) out of 10 treatment failures CPP decreased. This was associated with falls in SJO2 and mean velocity and rises in PI and AVDO2.

In conclusion, CPP is the most crucial parameter to monitor during ICP therapy. TCD and SJO2 recordings may complement CPP monitoring in identifying a lower threshold value for adequate CPP during therapy.

CAN DOCUMENTATION OF HEAD INJURIES IN ACCIDENT AND EMERGENCY DEPARTMENTS BE IMPROVED? RESULTS OF PILOT STUDIES USING A NEW HEAD INJURY PROFORMA RW Gullan, PO Byrne, S Wallace. Brook Hospital, London and Brighton

Head injury accounts for approximately $10 \%$ of all accident and emergency attendances. A comprehensive audit of "free hand" documentation of 158 head injuries in one of the three hospitals used in the survey demonstrated a poor level, or even a complete absence, of reference to what could be described as fundamental clinical details. A special proforma for documenting these patients has been designed to allow all aspects, including nursing notes, neurological observations, a simple diagnostic triage and management/treatment section, to be kept together on a single form. Adequate space for recording other injuries or opinions is available, thus avoiding separate records. Every effort has been made to make the input of data as easy as possible and mostly Yes/No or tick boxes are used, so that subsequent computerised data storage and easy audit would be possible in the future.

Two separate pilot studies (684 and 576 consecutive patients in each) have been undertaken and after the first, various recommendations incorporated, including the production of a form for children less than six years old. Some dramatic improvements in documentation (compared with "free hand" casualty notes) have been obtained: for instance, post traumatic amnesia recorded in $\mathbf{9 8 . 5 \%}$ of cases compared with $34 \%$ before; evidence of whether alcohol had recently been consumed or not recorded in $97 \%$ of cases as opposed to $43 \%$; and evidence of whether or not there was a compound injury in $89 \%$ as opposed to $10 \%$ previously recorded. At the completion of the second study, out of 22 Casualty Officers involved, only one was not in favour of the proforma being used on a permanent basis. The structure, design, problems and advantages of this form were discussed with reference to its use in 1260 patients in these two pilot studies.

IS HELMET USE BENEFICIAL TO MOTORCYCLISTS? VG Wagle, C Perkins, A Vallera. Hartford, CT, USA

This study included 83 motorcyclists, helmeted and nonhelmeted, involved in accidents. The majority of these patients were flown into a major trauma centre in the Northeast directly from the scene by a helicopter ambulance service (Life Star). The study showed that the majority of these patients were male, and under the age of 30 . Twenty seven had alcohol levels above the legal limit. Sixty nine per cent were nonhelmeted with a statistically significant greater number in this category with a Glasgow Coma Scale of 8 or under. Cervical spine injuries were discovered in only six patients, with none of the injuries occurring in the upper cervical region. Nine of the ten patients declared dead on arrival (DOA) were nonhelmeted.

In conclusion, helmets provided protection and did not increase the incidence of cervical spine injuries.

AN ANALYSIS OF BRITISH NEUROSURGICAL PRACTICE IN THE MANAGEMENT OF CHRONIC SUBDURAL HAEMATOMA

PO Byrne, JR Bartlett. Brook Hospital, London

Chronic subdural haematoma is a common condition which seems to be managed in a variety of ways with a relatively favourable prognosis. To get an idea of the frequencies of the various different approaches to management, a postal questionnaire was sent to every consultant neurosurgeon in the United Kingdom in the early part of 1991 . It was designed to examine the operative technique and postoperative management in patients with symptomatic chronic subdural haematoma warranting intervention. At attempt was also made to discover if there was an age limit or clinical state that would preclude treatment at all.

One hundred and twelve replies were received. Most respondents used one or more, usually two, burr holes and generally irrigated the cavity, often using a catheter; a quarter would sometimes leave in a drain. One used craniotomy as the main treatment. Postoperative bed rest was employed by half of respondents, usually for forty eight hours and not infrequently longer, with less than a 
fifth allowing immediate mobilisation. Most used clinical criteria to assess recurrence, though a quarter used CT scanning and three used needle aspiration. A quarter of respondents used steroids, fifteen using them regularly.

STEREOTACTIC RADIOFREQUENCY UPPER THORACIC SYMPATHECTOMY - AN ELEVEN YEAR EXPERIENCE HA Wilkinson. Worcester, Mass, USA

Between April 1979 and March 1991 the author performed 121 sympathectomies on 205 limbs in 91 patients using a percutaneous stereotactic radiofrequency technique, usually on an outpatient surgery basis Patient ages ranged from 10 to 81 years, with 41 males and 50 females. Four had had previous open surgical sympathectomy unsuccessfully. Patients suffered from hyperhidrosis, vascular occlusion, Raynaud's or other chronic vasculopathies, painful causalgia or reflex sympathetic dystrophy or Prinzmetals' angina.

The sympathectomy technique has evolved and is currently in its third phase. Changes were based on anatomical and clinical/radiographic correlations as well as careful patient follow up assessments. The modifications have reduced the frequency of early failures and seem to be reducing long term failures The present technique relies on neuroleptanalgesia with local anesthesia of superficia tissues only. Two 18 gauge radiofrequency "tic" needle electrodes are employed, with $10 \mathrm{~mm}$ bared tips for adults and $7 \mathrm{~mm}$ tips for children. A series of three lesions at 90 degrees for 180 seconds are made rostrocaudally at each of the ganglion sites selected. Lesion sites are based on C-arm fluoroscopy and an electrical output to produce a threshold greater than 1.0 volt. Lesion effectiveness is monitored by finger plethysmography and hand skin temperature measurement. Complications have generally not been severe except for symptomatic pneumothorax in some patients. Re-operation is easily performed when necessary.

\section{Epilepsy Surgery Symposium}

LONG TERM CONTROL OF EPILEPSY FOLLOWING TEMPORAL LOBECTOMY IN PATIENTS WITH LOW GRADE TEMPORAL TUMOURS

PJ Kirkpatrick, M Honvar, CE Polkey. Maudsley Hospital, London

Thirty seven patients (age range three-53 yrs) with tumour related temporal lobe seizures were presented. All had suffered chronic drug resistant temporal lobe seizures (mean age of onset $8 \cdot 1 \mathrm{yrs}$, range $0-30 \mathrm{yrs}$; mean duration $11 \cdot 1 \mathrm{yrs}$, range 2-39 yrs) and represented $20.5 \%$ of all cases undergoing temporal lobe resection for epilepsy over a 14 year period. Preoperative EEG tracings indicated localised epileptic foci in $89 \%$ of patients and of the $35 \mathrm{CT}$ scans performed $89 \%$ showed temporal lobe abnormalities including calcificaiton (47\%), low density lesions $(31 \%)$ and isolated temporal horn distortion (11\%). Electrocorticography (ECoG) was performed during 34 of the operations; $21(61 \%)$ showed an improved post-resection tracing. Tumour remvoal was microscopically incomplete in $70 \%$ and review of pathological specimens revealed a high incidence of dysembryoblastic neuroepithelial tumours. Postoperative mortality was zero, but one patient developed a permanent hemiplegia. Psychological assessment showed no measurable change in verbal and performance intelligence quotient following surgery, but a mild impairment of memory was detected in $51 \%$. Long term follow up (mean duration $5.6 \mathrm{yrs}$ ) indicated that $78 \%$ of patients were completely free of epilepsy. Relief of seizures could not be predicted by the intraoperative ECoG tracings nor by the early postoperative EEG findings which showed epileptiform phenomenon in $68 \%$. Outcome was also independent of tumour pathology and completeness of resection.

UNILATERAL TEMPORAL NEOCORTICECTOMY IN THE MANAGEMENT OF INTRACTABLE TEMPORAL LOBE EPILEPSY - LONG TERM OUTCOME AND NEUROPSYCHOLOGICAL SEQUELAE D McMackin, J Phillips, T Burke, H Staunton. Dublin

Temporal neocorticectomy had been carried out on over 70 patients at the Richmond Institute for Neurology and Neurosurgery, Dublin, since 1975 . This is a unique procedure among centres offering surgery for epilepsy in that excision is limited to the lateral neocortex whilst sparing all mesial structures.

Factors which predicted a good result were: 1) stereotyped onset of the seizure ( $p<$ $0.05)$; 2) unilateral antero-mid temporal spike ( $<<0.01)$; 3 ) greater volume of tissue removed at surgery $(p<0.05)$. On the basis of these predictive factors, a sub-category of patients was shown to have a $91 \%$ chance of having a favourable outcome from neocorticectomy. Overall outcome in the unselected series, show $60 \%$ of patients to be effectively seizure free.

The results of pre/post-operative neuropsychological evaluation suggested that the excision does not result in a deleterious effect on memory or cognition as measured by the Weschler Scales. Furthermore, although patients exhibit mild memory deficits related to the side of surgery, these are less severe than in patients from Montreal where deeper structures have been excised.

The authors recommended neocorticectomy as a conservative approach which can have maximum benefit in a carefully selected population.

1 McMackin D, Staunton H. Fournal of the Irish Colleges of Physicians and Surgeons 1991;20: 193-7.

MULTTPLE SUBPIAL TRANSECTION IN THE TREATMENT OF FOCAL MEDICALLY RESISTANT EPILEPSY

GP Kratimenos, DGT Thomas, SD Shorvon, D Fish. National Hospital, London

Based on experimental evidence suggesting that epileptogenic discharges require horizontal interaction of cortical neurons while cortical function depends on vertical fibre connections of the columnar units, the operation of multiple subpial transection was introduced some 20 years ago, as an attempt to relieve the medically intractable cases of focal epilepsy with epileptogenic foci located in functionally important cortical territories. The purpose of the transection of the horizontal intracortical fibres with preservation of the vertically oriented neural elements as wel as the penetrating blood vessels, was to reduce the occurrence of synchronised cell discharge without affecting the functional properties of the cortical tissue.

Four cases of focal epilepsy had been treated with multiple subpial transection during the last five years. In all cases the epileptogenic foci were located under or in cortical areas of major functional importance and therefore non-resectable. There were 2 male and 2 female patients with ages ranging from 21 to 33 years and with a history of medically intractable focal epilepsy of a mean duration of 12 years. All lesions were located under the motor cortex, two in the right and two under the left. Three of the patients had a radiologically identifiable structural lesion. Two of the lesions were biopsied sterotactically before the subpial transection confirming the preoperative diagnoses of low grade tumour. In the third case there was a previous history of cerebral abscess treated with aspiration 14 years before the current operation.

The functional postoperative results were excellent, although one of the cases was complicated by a subdural collection. The operation successfully controlled the epilepsy in only one case (the only one without structural abnormality), while from the remaining three cases only in one was an appreciable reduction of seizures detected.

From our limited experience conclusions suggest that the operation of multiple subpial transections may have a place in the surgical management of cases of focal epilepsy associated with non-resectable cortex and without an identifiable structural lesion. In those cases where lesions are present, the procedure could be combined with a stereotactic volumetric excision, as our cases indicate that in this context, subpial transection alone only poorly controls cortical spreading of epileptic discharges.

INTRAOPERATIVE TESTING OF MEMORY FUNCTION DURING HIPPOCAMPAL COOLING

KW Lindsay, $\mathbf{R}$ Duncan, $\mathbf{R}$ Gillham, D Cossar, D Sutton. Glasgow

Many centres perform the WADA test routinely before temporal lobectomy for intractable epilepsy in an attempt to identify the extent to which the hemisphere under test contributes to language and memory function. The production of complete amnesia when $\mathrm{Na}$ amytal is injected on the side of the proposed resection creates a major concern, but does not exclude the possibility of resective surgery as WADA testing carries a high incidence of false positives. In one such patient intraoperative memory testing was performed during hippocampal cooling using a specially designed probe. The patient was anaesthetised with propofol and alfentanil infusion and a laryngeal mask was used for airway control. Local anaesthetic was instilled around the craniotomy wound and at the sites of the headrest fixation. After completing the first stage of a right temporal resection and exposing the hippocampus, the propofol was discontinued and the laryngeal mask was removed. Memory testing remained intact before and after cooling the hippocampus to 23 degrees centrigrade, indicating the feasibility of proceeding to mesial resection. This was performed after reintroducing the propofol. Neuropsychological assessment in the immediate postoperative period detected no evidence of memory impairment. 
THE PLACE OF FORAMEN OVALE TELEMETRY IN PRESURGICAL EVALUATION OF PATIENTS WITH DRUG RESISTANT EPILEPSY

CE Polkey, CD Binnie. Maudsley Hospital, London

Videotelemetry with foramen ovale electrodes was first introduced by Wieser as a means of demonstrating the onset of partial complex seizures in the mesial temporal structures. Since 1987 over 130 patients had been investigated using this method of recording.

The electrodes were inserted under general anaesthesia and with radiographic control. In the first eighty or so patients single contact wires were inserted through needles used for thermocoagulation introduced through the foramen ovale by the usual method. In the remaining patients multi-contact electrodes were inserted through splittable cannualae. All patients received antibiotic cover whilst the electrodes were in place. Recordings were carried out for periods of up to ten days with anticonvulsant withdrawal, sleep deprevation and close supervision.

The method proved reasonably safe, the youngest patient investigated was five years old. There was no mortality and no major neurological morbidity. Three patients had proven meningitis, one from an unsuccessful insertion; all were rapidly controlled with intravenous antibiotics. A number of patients had numbness in the divisions of the fifth nerve, in proportion to the difficulty of insertion, but all recovered. In one patient the electrodes had clearly entered brain substance and were removed with 24 hours and the patient sucessfully reimplanted three months later.

HIPPOCAMPAL VOLUMETRIC STUDIES AND SURGICAL TREATMENT OF TEMPORAL LOBE EPILEPSY

MJ Cook, DR Fish, SD Shorvon, JM Stevens. Queen Square and St Mary's Hospitals, London

Determination of structural lesions on imaging studies correlates strongly with postoperative seizure prognosis in epilepsy surgery. Temporal lobe pathology is most frequently Ammons Horn sclerosis (AHS), and several different imaging techniques have been utilised in detection of this with varying success. MRI using a $3 \mathrm{D}$ volume technique had been used which allowed total hippocampal volume to be measured and symmetry evaluated. Hippoccampal surface area was calculated in sequential $1.5 \mathrm{~mm}$ thick contiguous images, using a IGE IC workstation. Total volumes were calculated and surface area versus hippocampal length plotted, permitting morphometric analysis of the hippocampus. Regional involvement and extent of volume loss were then assessed. Previous studies have all been significantly limited by thick slices and large inter slice gap, and have not provided this detailed information. Patients with hippocampal and extra-hippocampal pathology were studied, and volumes compared with normal values derived from 10 neurologically normal controls. Hippocampal volume loss was demonstrated in patients with clinically typical temporal lobe epilepsy, who did not have extra-hippocampal lesions. Hippocampal volume loss did not occur in patients with extra-temporal or temporal neocortical lesions.

Volumetric analysis of the hippocampus can demonstrate asymmetry, regional involvement, and distinguish between hippocampal and extra-hippocampal pathologies.

PHASE II PRESURGICAL EVALUATION OF COMPLEX PARTIAL SEIZURES WITH DEPTH ELECTRODES

W Harkness, M Levesque. Los Angeles, California, USA

Thirty one patients with complex partial seizures had phase II presurgical evaluation with stereotactically implanted electrodes (SEEG) between January 1988 and December 1990 at UCLA medical center. There were 20 males and 11 females in this group with a mean age of 30 years (range 16-48). Mean age at seizure onset was 14 years and the mean delay to phase II evaluation was 16 years. Five patients had both depth electrode implantation and insertion of subdural strips in the same procedure $(16 \%)$. There were four clinical complications of electrode implantation (12.9\%) and no mortality. One patient required early electrode deplant before seizure activity could be recorded on SEEG. As a result of the phase II data 17 patients had surgery to remove the identified epileptogenic focus. En bloc temporal lobectomy was carried out in 14 cases, a selective amygdalohippocampectomy in 2 cases and an extratemporal resection in 1. Fourteen patients failed to proceed to surgery following phase II. Three cases had seizures localised to a mesial temporal focus but did not have surgery for other reasons, while five had bitemporal seizure onset and five had extratemporal, diffuse or nonlocalised seizure onset. The phase I data for all cases was reviewed and analysed in an attempt to identify factors that would suggest previous to phase II that the patients would not be surgical candidates, thus preventing unneccessary invasive recordings.

APPLICATION OF THE G-T LOCALISER FOR DEPTH ELECTRODE INSERTION IN THE INVESTIGATION OF DRUG RESISTANT EPILEPSY

GP Kratimenos, DGT Thomas, SD Shorvon, DR Fish. National Hospital, London

Intracerebral insertion of electrodes for electroencephalographic recording provides an accurate spatiotemporal localisation of the epileptic activity during the pre-operative evaluation of patients with intractable epilepsy not otherwise localised. The localising value of the method depends on the precise and predetermined placement of the electrodes and for this, a stereotactic technique of insertion based on information derived from computerised imaging (CT and MRI) and sterotactic angiography is highly desirable.

Various methods of multimodal stereotactic image integration have been described. A common limitation is the serial performance of the imaging studies following the stereotactic frame application or if the frame is to be removed between studies, localising pins penetrating the outer skull table must remain in place. Computerised analysis and image integration is an inherent part of the described methods and the operation takes place immediately following the target definition.

An alternative method of stereotactic aquisition of multimodal image information was presented which uses the G-T stereotactic relocatable localiser. The temporal freedom provided during the investigation-operation period could potentially be advantageous offering an unhurried multi-image integration and targetting in any individual case combined with less discomfort for the patient. The accuracy of the system is well proven and the localising value of the technique is excellent as the presenting cases suggest. There were no complications in any of the patients included in the study although the recording period extended in some cases as long as 18 days. The accuracy of the electrode placement was easily verified during the post-operative period using standard radiographic and MRI studies and this information added to the localising value of the technique. 